\title{
The Blind Men and the Elephant as a Metaphor for Surface Analysis, as Applied to the Preparation and Analysis of New, Highly Stable Materials for Separations Science
}

Matthew R. Linford, ${ }^{1}$ Sarah Copeland, ${ }^{1}$ Andrew Dadson, ${ }^{2}$ Robert C. Davis, ${ }^{3}$ David Jensen, ${ }^{1}$ Rebecca Olsen, ${ }^{1}$ Gaurav Saini, ${ }^{1}$ V. Shutthanandan, ${ }^{4}$ Jun Song, ${ }^{3}$ Michael A. Vail, ${ }^{2}$ Richard Vanfleet, ${ }^{3}$ Landon A. Wiest, ${ }^{1}$ Richard D. Wyman, ${ }^{3}$ Li Yang ${ }^{1}$

${ }^{1}$ Department of Chemistry and Biochemistry, Brigham Young University, Provo, UT 84602, ${ }^{2}$ US Synthetic Corporation, Orem, UT, ${ }^{3}$ Department of Physics and Astronomy, Brigham Young University, Provo, UT 84602, ${ }^{4}$ EMSL, Environmental Molecular Sciences Laboratory, Pacific Northwest National Laboratory, Richland, WA 99352

Most of us heard the story of the blind men and the elephant as children. In this old tale from India each man in a group of blind men touches a different part of an elephant. Each walks away with a different experience and subsequently argues that the elephant is like a spear (the tusk), a thick rope (the trunk), a wall (the flank), etc. Only the combination of their stories would have provided a complete, or at least more complete, picture of what an elephant really is. In some sense this is the story of surface analysis, which lacks a single analytical tool that can provide comprehensive information about a surface or interface. We rely on X-ray photoelectron spectroscopy (XPS) for surface elemental analysis and oxidation state information, spectroscopic ellipsometry for film thicknesses and optical constants, contact angle measurements to understand surface wetting, Fourier transfer infrared spectroscopy (FTIR) to reveal functional group information, negative and positive ion time-of-flight secondary ion mass spectrometry (ToF-SIMS)to provide molecular fragments and trace element detection, Rutherford backscattering (RBS) for elemental composition and atom distributions in moderately thick films (typically at least a few nanometers), nuclear reaction analysis (NRA) for absolute quantitation of atomic compositions of thin films, atomic force microscopy (AFM) for surface roughness, scanning electron microscopy (SEM) to reveal surface features and patterning, BET (Brunauer, Emmett, Teller) isotherm measurements to provide surface areas and pore sizes, etc. Combining such information typically provides the most complete view of a surface or interface. The purpose of my talk is to discuss a problem that illustrates the importance of using multiple analytical methods to better understand surfaces and interfaces - an important conclusion of my talk is that no single instrument could have provided the insight into the problem that was gained from the combination of techniques. In particular, we are currently developing and/or modifying highly stable materials based on diamond, zirconia, and/or graphite, which can withstand extreme $\mathrm{pH}$ values, temperatures, and/or other harsh chemical conditions, as stationary phases or supports for liquid chromatography.1-3At present this is an important topic in separations science - about 
$70 \%$ of the analytes separated by pharmaceutical companies are basic, and these separations would often benefit from being run at high $\mathrm{pH}$ values $(>10)$. Also, to reduce mobile phase viscosities, which lowers back pressures, and to improve mass transfer, it is increasingly common to perform HPLC (and UPLC) at elevated temperatures (as high as $200^{\circ} \mathrm{C}$ ). To develop materials that can withstand these harsh conditions, we have recently prepared core-shell particles made from $c a$. 1.5 micron diamond or zirconiacores that havemultilayers of nanodiamond and poly(allylamine) on their surfacesin the form of a high porosity shell. After making the particles they are functionalized with $\mathrm{C}_{18}$ chains. Some of this chemistry has also been performed on planar surfaces, which increases the number of analytical tools available to study the problem. An understanding of our new materials has come by combining information gleaned from SEM (including cross sectional analyses prepared by ion milling that show the core-shell nature of the particles), RBS (which clearly shows layerby-layer (LbL) growth over a model silicon substrate), XPS and positive and negative ion ToF-SIMS (which show the expected chemical transformations in different ways), FTIR (which shows the expected functional groups in increasing concentrations during the LbL process), wetting (which shows, among other things, the addition of alkyl chains to form the $\mathrm{C}_{18}$ phase on model planar surfaces), and BET isotherm measurements (which show that our materials have reasonably high surface areas and $c a .20 \mathrm{~nm}$ pores).Chromatography (separations) has been performed on these materials, which in and of itself is a type of surface characterization.

Like the story of the blind men each sampling a different part of the elephant, a complete picture of our new materials only comes from a combination of analytical

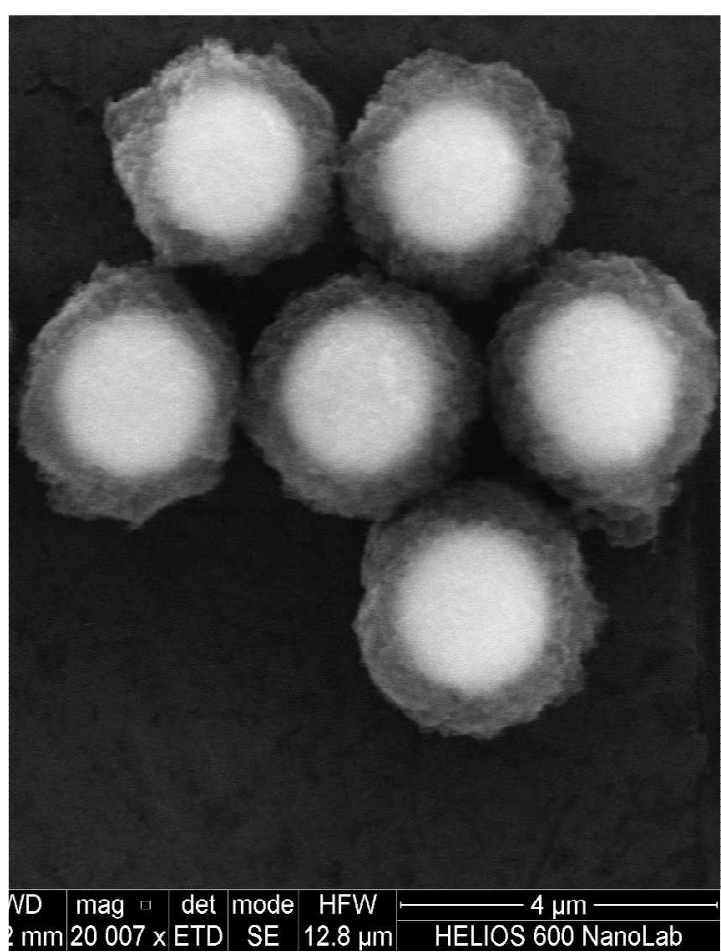

Figure 1. SEM image of core-shell particles consisting of a $\mathrm{ZrO}_{2}$ core and a poly(allylamine)-nanodiamond shell. techniques.

\section{References}

1. Saini, G.; Jensen, D.S.; Wiest, L.A. Vail, M.A.; Dadson, A.; Lee, M.L.; Shutthanandan, V.; Linford, M.R. Functionalized Core-Shell Diamond as a Porous Support/Stationary Phase for Solid Phase Extraction and High Performance Liquid Chromatography. Submitted.

2. Saini, G.; Wiest, L.A.; Herbert, D.; Biggs, K.N.; Dadson, A.; Vail, M.A.; Linford, M.R. $\mathrm{C}_{18}, \mathrm{C}_{8}$, and perfluoro reversed phases on diamond for solid-phase extraction. J. Chrom. A. 2009; 1216, 3587-3593.

3. Saini, G.; Yang, L.; Lee, M.L.; Dadson, A.; Vail, M.A.; Linford, M.R. Amino-Modified Diamond as a Durable Stationary Phase for Solid-Phase Extraction. Analytical Chemistry2008; 80, 62536259. 